\title{
Morfologia das fibras musculares esqueléticas de frangos de corte de diferentes linhagens criados em sistemas de confinamento e semiconfinamento ${ }^{1}$
}

\author{
Luciene Aparecida Madeira ${ }^{2}$, José Roberto Sartori ${ }^{3}$, Érika Salgado Politi Braga Saldanha ${ }^{4}$, \\ Carla Cachoni Pizzolante ${ }^{4}$, Maeli Dal Pai Silva ${ }^{5}$, Ariel Antonio Mendes ${ }^{6}$, Sabrina Endo \\ Takahashi $^{2}$, William Vicent Narvaez Solarte ${ }^{7}$
}

\footnotetext{
1 Parte da dissertação de Mestrado apresentada ao Programa de Pós-Graduação em Zootecnia da FMVZ, UNESP, Campus de Botucatu, $S P$, pela primeira autora. Apoio: CAPES.

2 Doutoranda do Programa de Pós-Graduação em Zootecnia da FMVZ, Universidade Estadual Paulista, Campus de Botucatu, SP.

${ }^{3}$ Departamento de Melhoramento e Nutrição Animal, FMVZ, Universidade Estadual Paulista, Campus de Botucatu, SP.

${ }^{4}$ APTA, Unidade de Pesquisa e Desenvolvimento de Brotas, SP.

5 Departamento de Morfologia, IBB, Universidade Estadual Paulista, Campus de Botucatu, SP.

${ }^{6}$ Departamento de Produção e Exploração Animal, FMVZ, Universidade Estadual Paulista, Campus de Botucatu, SP.

7 Universidade de Caldas, Colombia.
}

RESUMO - Avaliou-se o efeito da linhagem, do sistema de criação e do sexo sobre o peso vivo, o rendimento de carcaça e de pernas e os aspectos morfológicos das fibras musculares esqueléticas do músculo flexor longo do hálux de frangos de corte. O delineamento experimental foi inteiramente casualizado, em esquema fatorial 4 x 2 x 2, ou seja, quatro linhagens (Ross308, Pescoço Pelado Label Rouge, Caipirinha e Paraíso Pedrês), dois sistemas de criação (confinamento e semiconfinamento) e dois sexos, com duas repetições por tratamento, sendo que cada ave retirada ao acaso aos 56 dias de idade foi considerada uma unidade experimental, totalizando 64 aves. A linhagem Ross apresentou maior peso vivo e maiores pesos de carcaça, de pernas, de carne de penas e do músculo flexor longo do hálux e maiores rendimentos de carcaça e de carnes de pernas que as outras linhagens. A maior massa muscular das aves selecionadas para alta taxa de crescimento está relacionada ao aumento na área dos três tipos de fibras musculares (SO, FOG e FG). Machos apresentaram maior massa muscular e musculatura mais glicolítica que fêmeas. O sistema de semiconfinamento alterou a composição de fibras musculares esqueléticas dos machos, tornando-a mais oxidativa, porém, esse efeito não foi observado nas fêmeas.

Palavras-chave: exercício, fibra muscular, frangos de corte, linhagem, semiconfinamento

\section{Morphology of skeletal muscle fibers of different broiler chicken strains bred in confined and semi-confined systems}

\begin{abstract}
The effect of strain, breeding system and sex on body weight, carcass and leg yield, and morphologic aspects of skeletal muscle fibers of flexor hallucis longus muscle of broiler chickens was evaluated in this study. The experiment consisted of a 4x2x2 factorial arrangement of treatments, with four strains (Ross-308, Naked Neck Label Rouge, Caipirinha and Paraiso Pedrês), two breeding systems (confinement and semi-confinement) and two sexes, with two replicates per treatment, considering each bird selected randomly at 56 days of age an experimental unit, with a total of 64 birds. Ross strain had the highest values of body weight, weights of legs and meat of legs and weight of flexor hallucis longus muscle and highest values of carcass yield and meat of leg yield. The higher muscle mass of birds selected for high growth is related to an increase in the area of the three types of muscle fibers evaluated (SO, FOG and FG). Males presented higher muscle mass and a musculature higher in fast-glycolityc-type fibers than females. Semi-confined system modified the composition of skeletal muscle fibers of males, increasing the oxidative-type fibers. However this effect was not observed in females.
\end{abstract}

Key Words: broiler chickens, exercise, muscle fiber, semi-confined, strain

\section{Introdução}

A carne de frango, seguindo a tendência mundial, tem sido largamente consumida no Brasil. A população tem procurado produtos diferenciados, como carne de ave mais avermelhada e firme. Este tipo de produto, com sabor mais pronunciado, é característico de animais mais velhos, em idade próxima à maturidade sexual (Bastianelli, 2001), de aves que não sofreram melhoramento genético intenso (linhagens caipiras) ou de aves submetidas a exercício (criação extensiva ou em semic onfinamento). 
Para atender esse mercado, foram criadas várias linhagens coloniais, como a Pescoço Pelado Label Rouge, de origem francesa; Embrapa 041, produzida pelo Centro Nacional de Pesquisa em Suínos e Aves da Embrapa, Concórdia - SC; a Paraíso Pedrês, criada pela Granja Aves do Paraíso, Itatiba - SP, e a linhagem Caipirinha, produzida pela ESALQ/USP, em Piracicaba - SP (Takahashi, 2003).

Aproximadamente 30 a $40 \%$ do peso vivo animal consiste de músculos esqueléticos (Pearson \& Young, 1989). Entre esses músculos, destacam-se o vermelho, constituído predominantemente por fibras oxidativas, e o branco, formado predominantemente por fibras glicolíticas (Banks, 1992).

Nos mamíferos, assim como nas aves, as fibras musculares podem ser identificadas, com base nas suas características metabólicas e contráteis, em três tipos: tipo I - de contração lenta e oxidativa (SO), tipo IIA - de contração rápida e oxidativa (FOG); e tipo IIB - de contração rápida e glicolítica (FG) (Peter et al., 1972; Banks, 1992).

Para o entendimento do desenvolvimento muscular, é fundamental estudar os mecanismos que regulam o número, o tipo e o tamanho das fibras musculares, relacionados a vários fatores que interferem no desenvolvimento muscular nos períodos pré e pós-natal (Dauncey \& Gilmour, 1996), como os fatores intrínsecos (genética, fatores reguladores de crescimento, ativadores de transcrição, status endócrino, proteinases musculares e inervação) e os ambientais (dieta e temperatura ambiente), a atividade motora, os agentes repartidores de nutrientes, a idade, o sexo, as doenças, o tipo de músculo e a localização das fibras no músculo (Gonzales \& Sartori, 2002).

O melhoramento genético do frango de corte para maior massa muscular e rapidez de crescimento reduz a capacidade oxidativa da musculatura dos frangos de corte, resultando em músculos mais anaeróbios (Soike \& Bergmann, 1997).

O exercício, em aves, pode levar a certas mudanças adaptativas na capacidade oxidativa dos músculos esqueléticos, similares às observadas no homem e em outros mamíferos. Em frangos de corte, o exercício resulta em alterações no tamanho dos músculos e na modulação das suas fibras, confirmando que o padrão da atividade influencia na diferenciação do músculo. Tanto a colocação de barreiras quanto de rampas nos boxes convencionais de frangos de corte foram determinantes para obtenção de músculos mais pesados após 3 a 4 semanas de exposição à nova situação (Khaskiye et al., 1987; Sandusky \& Heath, 1988). O sistema de criação em semiconfinamento, em comparação ao de confinamento, permite que as aves se movimentem continuamente e por maiores distâncias e, dependendo da intensidade e duração do exercício, incide em alterações significativas na musculatura esquelética das aves.

Neste trabalho foram avaliados os efeitos da linhagem, do sistema de criação e do sexo sobre o peso vivo, o rendimento de carcaça e de pernas e os aspectos morfológicos das fibras musculares esqueléticas do músculo flexor longo do hálux de frangos de corte.

\section{Material e Métodos}

O experimento foi conduzido nas instalações experimentais da Unidade de Pesquisa e Desenvolvimento de Brotas, da Secretaria da Agricultura e do Abastecimento do Estado de São Paulo, no período de 17 de fevereiro a 13 de abril de 2003.

Foram criados 1.600 pintinhos de corte, distribuídos em um delineamento experimental inteiramente casualizado, em esquema fatorial 4 × 2 × 2 , ou seja, quatro linhagens (Ross308, Pescoço Pelado Label Rouge, Caipirinha e Paraíso Pedrês), dois sistemas de criação (confinamento e semiconfinamento) e dois sexos (macho e fêmea), com duas repetições por tratamento.

As aves foram alojadas em galpão experimental de alvenaria coberto com telhas de barro (pé-direito de 2,80 m), dividido em boxes com 5,0 $\mathrm{m}^{2}$ cada um. Os boxes permitiam acesso a áreas de piquetes gramados (Brachiaria decumbens), cercadas com arame tipo tela trançado revestido com sombrite. As aves criadas no sistema de semiconfinamento tiveram acesso ao piquete gramado ( $3 \mathrm{~m}^{2} /$ ave $)$ durante o dia, sendo recolhidas ao boxe à noite.

No alojamento, após a pesagem inicial, os pintos foram distribuídos em 32 boxes previamente sorteados e receberam vacina contra coccidiose ministrada na ração. Aos 15 dias de idade, foram vacinadas contra bouba aviária.

Cada boxe foi equipado com um comedouro inicial (cone de pressão), um bebedouro pendular e uma campânula elétrica provida de lâmpada infravermelha. Após a primeira semana de idade, os comedouros iniciais foram substituídos por comedouros tubulares e, aos 14 dias de criação, as campânulas foram retiradas.

A partir dos 35 dias de idade, as aves criadas em semiconfinamento tiveram acesso aos piquetes até o final do experimento.

A ração e a água foram fornecidas à vontade durante todo o período de criação e o programa de alimentação foi dividido, segundo recomendações de Figueiredo (2000), em duas fases (Tabela 1): ração inicial (1 a 28 dias) e ração de crescimento (29 a 56 dias). 
Tabela 1 - Composição das rações segundo a fase de criação Table 1 - Diet composition according to the breeding phase

\begin{tabular}{|c|c|c|}
\hline $\begin{array}{l}\text { Composição } \\
\text { Composition }\end{array}$ & $\begin{array}{l}1 \text { a } 28 \text { dias } \\
1-28 \text { days }\end{array}$ & $\begin{array}{l}29 \text { a } 56 \text { dias } \\
29-56 \text { days }\end{array}$ \\
\hline Milho & 60,916 & 66,570 \\
\hline Corn & & \\
\hline $\begin{array}{l}\text { Farelo de soja } \\
\text { Soybean meal }\end{array}$ & 34,547 & 29,084 \\
\hline Fosfato bicálcico & 1,869 & 1,738 \\
\hline $\begin{array}{l}\text { Dicalcium phosphate } \\
\text { Calcário calcítico } \\
\text { Limestone }\end{array}$ & 1,062 & 1,187 \\
\hline $\begin{array}{l}\text { Óleo de soja } \\
\text { Soybean oil }\end{array}$ & 0,763 & 0,572 \\
\hline Sal & 0,350 & 0,350 \\
\hline $\begin{array}{l}\text { Salt } \\
\text { Dl-metionina }\end{array}$ & 0,093 & 0,099 \\
\hline DL-methionine & & \\
\hline Suplemento vitamínico ${ }^{1}$ & 0,100 & 0,100 \\
\hline $\begin{array}{l}\text { Supplement vitamin } \\
\text { Suplemento mineral }\end{array}$ & 0,100 & 0,100 \\
\hline $\begin{array}{l}\text { Supplement mineral } \\
\text { Simbiótico plus }{ }^{\circledR 3} \\
\text { Simbiotic plus }\end{array}$ & 0,200 & 0,200 \\
\hline Total & 100,0 & 100,0 \\
\hline
\end{tabular}

Valor calculado

Calculated value

\begin{tabular}{lcc}
\hline $\mathrm{EM}, \mathrm{kcal} / \mathrm{kg}$ & 2.800 & 2.900 \\
$\begin{array}{l}M E, k c a l / k g \\
\mathrm{~PB}, \%\end{array}$ & 20,00 & 18,00 \\
$\begin{array}{l}\text { L } \\
\text { Lisina, \% } \\
\text { Lysine }\end{array}$ & 1,00 & 0,88 \\
$\begin{array}{l}\text { Metionina, \% } \\
\begin{array}{l}\text { Methionine } \\
\text { Ca, \% }\end{array}\end{array}$ & 0,43 & 0,41 \\
$\begin{array}{l}\text { P disponível, \% } \\
\text { Available P }\end{array}$ & 1,00 & 1,00 \\
& 0,46 & 0,43
\end{tabular}

${ }^{1}$ Suplemento vitamínico, níveis de garantia por kg do produto (Supplement vitamin, garanty levels per $\mathrm{kg}$ of the product): vit. A - 1.500.000 UI; vit. D3 $500.000 \mathrm{UI}$; vit. E - $3.000 \mathrm{mg}$; vit. K3 - $200 \mathrm{mg}$; tiamina (tiamine) - $250 \mathrm{mg}$; riboflavina (riboflavine) - $1.125 \mathrm{mg}$; piridoxina (pyridoxine) - $375 \mathrm{mg}$; vit. B12 - $3.000 \mathrm{mg}$; niacina (niacin) - $7.500 \mathrm{mg}$; pantotenato de cálcio (calcium pantothenate) - $2.500 \mathrm{mg}$; ácido fólico (folic acid) - $1.375 .5 \mathrm{mg}$; biotina (biotin - $12,5 \mathrm{mg}$; cloreto de colina (choline choride) - $81.250 \mathrm{mg}$; metionina (methionine) - $325.000 \mathrm{mg}$; antioxidante (antioxidant) - $5.000 \mathrm{mg}$.

2 Suplemento mineral (mineral supplement): $\mathrm{Fe}-5.000 \mathrm{mg}$; $\mathrm{Cu}-70.000 \mathrm{mg} ; \mathrm{Mn}$ $60.000 \mathrm{mg}$; Zn - $50.000 \mathrm{mg}$; I - $1.250 \mathrm{mg}$; Se - $200 \mathrm{mg}$.

${ }^{3}$ Simbiótico plus ${ }^{\circledR}-10^{6}$ UFC de Enterococcus sp. por grama do produto e $85 \%$ de mananoligossacarídeos.

${ }^{3}$ Simbiotic plus ${ }^{\circledR}-10^{6}$ UFC of Enterococcus sp. per grams of product in $85 \%$ and mananoligossacarid.

As rações foram produzidas na forma farelada na Fábrica de Rações da FMVZ, UNESP, Campusde Botucatu, SP, sendo isentas de promotores de crescimento, antibióticos, anticoccidianos e ingredientes de origem animal. Entretanto, foram acrescidas, para ambas as fases, de Simbiótico plus ${ }^{\circledR}$ - BioCamp Laboratórios Ltda., produto composto por probiótico e prebiótico.

Na fase final de crescimento, aos 56 dias de idade, foram retiradas ao acaso duas aves por boxe (quatro por tratamento, em um total de 64 aves), que foram abatidas no Abatedouro
Experimental da FMVZ, UNESP, Campus de Botucatu, SP, após jejum por 8 horas. Os cálculos derendimento de carcaça e das partes foram feitos com base no peso vivo e no peso da carcaça. $\mathrm{O}$ peso vivo foi determinado individualmente na plataforma, antes do abate, e o peso de carcaça, pela pesagem da ave eviscerada, sem pescoço, pernas e gordura abdominal. Foram avaliados os peso de peito, pernas (coxa e sobrecoxa), dorso, asas, carne de coxae carne de peito, segundo metodologia descrita por Mendes et al. (1993).

O músculo flexor longo do hálux da perna direita foi dissecado imediatamente após o abate e pesado sem tendões e sem gordura. Em seguida, foram realizadas secções transversais na região mediana do músculo para retirada de amostras, as quais foram imediatamente congeladas em $\mathrm{N}$-Hexana, previamente resfriada a $-156^{\circ} \mathrm{C}$ em nitrogênio líquido, durante dois minutos (Chayen et al., 1969). Seqüencialmente, foram identificadas e acondicionadas em botijão de nitrogênio líquido para análises histoenzimológicas realizadas no Laboratório de Histoenzimologia do Departamento de Morfologia do IBB, UNESP, Campus de Botucatu, SP.

A escolha do músculo flexor longo do hálux baseou-se nos relatos de Sartori et al. (2001), que, em estudo com frangos de corte Ross aos 42 dias de idade, verificou que o músculo flexor longo do hálux, pela sua composição de tipos de fibras (25,33\% de SO, 53,29\% de FOG e $23,55 \%$ de FG), por seu tamanho e sua fácil localização e identificação, pode ser utilizado nos estudos da musculatura esquelética de frangos de corte.

Os fragmentos congelados de tecido muscular foram transferidos para a câmara de um micrótomo criostato Reichert-young a $-20^{\circ} \mathrm{C}$ e mantidos neste ambiente por 45 minutos. Após esse tempo, foi fixado perpendicularmente no suporte metálico do criostato utilizando-se resina especial Tissue Tek, OCT - Optimal Critical Temperature Compound. Em seguida, foram obtidas várias séries de cortes histológicos (10 $\mathrm{mm}$ de espessura), os quais foram colhidos em lâminas mantidas à temperatura ambiente, conforme procedimento de Eversole \& Standish (1970).

Os cortes foram submetidos às seguintes técnicas de coloração: Hematoxilina-Eosina (HE), para análise da morfologia das fibras musculares; Nicotinamida Adenina Dinucleotídeo Tetrazólio Redutase (NADH-TR), para diferenciar a atividade metabólica das fibras em oxidativa e/ou glicolítica e ATPase miofibrilar (m-ATPase) após préincu bação em meio ácido ( $\mathrm{pH} 4,6$ ), que indica a velocidade de contração das fibras, conforme metodologia de Dubowitz \& Brooke (1984). A comparação destas duas técnicas possibilita a diferenciação dos tipos de fibras, conforme os critérios determinados por Peter et al. (1972), e sua classi 
ficação em SO (Slow Oxidative), FOG (Fast Oxidative Glycolytic) e FG (Fast Glycolytic).

O tamanho da fibra foi avaliado pela medida da área (Dubowitz \& Broke, 1984). Para cálculo da freqüência e do tamanho dos tipos de fibras, foram analisados os cortes que reagiram com a m-ATPase após pré-incubação ácida ( $\mathrm{pH} 4,6$ ), obtendo-se a diferenciação dos três tipos de fibras. Dos cortes presentes em cada lâmina, foram escolhidos o mais íntegro e aquele com melhor padrão de reação. As reações NADH-TR serviram como controle para a identificação dos tipos de fibras.

Para contagem, estabelecimento das freqüências (\%) e obtenção do diâmetro dos tipos de fibras, foram analisados por corte dez campos microscópicos utilizando-se microscópio ótico comum acoplado ao computador e ao sistema de análise de imagens da Leica (Image-Pro Plus versão 4.5.0.27). Foram contadas e medidas as fibras constantes nos campos microscópicos.

Os dados foram submetidos à análise de variância (ANOVA) com auxílio do procedimento GLM do programa SAS (1996), aplicando-se o teste Tukey a 5\% de probabilidade para verificar diferenças significativas entre as médias dos tratamentos.

\section{Resultados e Discussão}

Não houve interação significativa $(\mathrm{P}>0,05)$ sistema de criação $\times$ linhagem $\times$ sexo para as variáveis analisadas na carcaça (peso e rendimentos de carcaça, de pernas e de carne de pernas e morfologia das fibras musculares do músculo flexor longo do hálux). Não foram observados efeitos das interações $(\mathrm{P}>0,05)$ sistema de criação $\times$ linhagem e sistema de criação $\times$ sexo sobre o peso vivo e o peso da carcaça (Tabela 2). A interação linhagem $\times$ sexo, no entanto, foi significativa $(\mathrm{P}<0,05)$ para peso de carcaça $\mathrm{e}$ seu desdobramento é demonstrado na Tabela 3.

Dentro do fator sexo, a linhagem afetou significativamente $(\mathrm{P}<0,05)$ o peso da carcaça (Tabela 3$)$, de modo que, entre os machos, aves Ross foram superiores (3.387 e $2.447 \mathrm{~g}$ ) às da linhagem Paraíso Pedrês (2.250 e $1.497 \mathrm{~g}$ ). As linhagens Caipirinha (1.723 e 1146 g) e Pescoço Pelado (1.750 e $1.178 \mathrm{~g})$ não diferiram e apresentaram resultados inferiores para peso de carcaça quando comparadas às Paraíso Pedrês e Ross. Entre as fêmeas, os maiores $(\mathrm{P}<0,05)$ pesos de carcaça $(2.734 \mathrm{e} 1.989 \mathrm{~g})$ foram obtidos nas aves Ross em comparação às Paraíso Pedrês (1.794 e 1.201 g), seguidas das aves Caipirinha (1.297 e $851 \mathrm{~g}$ ) e das Pescoço Pelado (1.144 e $762 \mathrm{~g}$, respectivamente). Os resultados deste estudo corroboram a afirmação de Silva et al. (2001)
Tabela 2 - Pesos médios (gramas) de carcaça (CA), pernas (PE), carne de pernas (CPE) e do músculo flexor longo do hálux (MFLH) de frangos de corte aos 56 dias de idade, segundo a linhagem, o sexo e o sistema de criação

Table 2 - Mean values (gram) of weight carcass (CA), legs (PE), meat of legs (CPE) and flexor hallucis longus muscle (MFLH) of broiler chickens at 56 days of age according to strain, sex and system

\begin{tabular}{lllll}
\hline & \multicolumn{5}{c}{$\begin{array}{c}\text { Peso (gramas) } \\
\text { Weight, grams }\end{array}$} \\
\cline { 2 - 5 } & CA & PE & CPE & MFLH \\
\hline $\begin{array}{l}\text { Linhagem (L) } \\
\text { Strain }\end{array}$ & 0,01 & 0,01 & 0,01 & 0,01 \\
\hline $\begin{array}{l}\text { Ross } \\
\text { Paraíso Pedrês }\end{array}$ & $1349 \pm 170$ & $460 \pm 64$ & $296 \pm 44$ & $1,47 \pm 0,36 \mathrm{~b}$ \\
Caipirinha & $998 \pm 167$ & $340 \pm 62$ & $217 \pm 41$ & $1,10 \pm 0,23 \mathrm{c}$ \\
Pescoço Pelado & $970 \pm 238$ & $329 \pm 88$ & $214 \pm 58$ & $0,99 \pm 0,32 \mathrm{c}$ \\
\hline $\begin{array}{l}\text { Sexo (S) } \\
\text { Sex }\end{array}$ & 0,01 & 0,01 & 0,01 & 0,01 \\
\hline $\begin{array}{l}\text { Macho } \\
\text { Male }\end{array}$ & $1.567 \pm 530$ & $532 \pm 169$ & $352 \pm 125$ & $1,67 \pm 0,56 \mathrm{a}$ \\
Fêmea & $1.201 \pm 496$ & $385 \pm 141$ & $253 \pm 101$ & $1,26 \pm 0,61 \mathrm{~b}$ \\
Female & & & & \\
\hline
\end{tabular}

Sistema de criação (C)

$\begin{array}{lllll}\text { Breeding system } & \mathrm{ns} & \mathrm{ns} & \mathrm{ns} & \mathrm{ns}\end{array}$

Confinamento $\quad 1.371 \pm 538 \quad 453 \pm 170 \quad 307 \pm 123 \quad 1,48 \pm 0,63$

Confined

Semiconfinamento $\quad 1.363 \pm 544 \quad 453 \pm 171 \quad 299 \pm 122 \quad 1,44 \pm 0,61$

Semi-confined

\begin{tabular}{|c|c|c|c|c|}
\hline Interação $\mathrm{L} \times \mathrm{S}$ & 0,05 & 0,01 & 0,01 & $\mathrm{~ns}$ \\
\hline $\begin{array}{l}L \times S \text { interaction } \\
\text { Interação } \mathrm{L} \times \mathrm{C}\end{array}$ & $\mathrm{ns}$ & ns & $\mathrm{ns}$ & ns \\
\hline $\begin{array}{l}L \times C \text { interaction } \\
\text { Interação } \mathrm{C} \times \mathrm{S} \\
C \times S \text { interaction }\end{array}$ & $\mathrm{ns}$ & ns & 0,05 & $\mathrm{~ns}$ \\
\hline $\begin{array}{l}\text { Interação } \mathrm{L} \times \mathrm{S} \times \mathrm{C} \\
L \times S \times C \text { interaction }\end{array}$ & $\mathrm{ns}$ & $\mathrm{ns}$ & $\mathrm{ns}$ & $\mathrm{ns}$ \\
\hline CV (\%) & 6,98 & 7,18 & 9,12 & 16,79 \\
\hline
\end{tabular}

a, b Médias na coluna seguidas de letras distintas diferem $(P<0,05)$ pelo teste Tukey.

a, b Means in a column followed by different letters differ $(P<0.05)$ by Tukey test.

de que as linhagens coloniais Caipirinha e Pescoço Pelado são de crescimento lento, enquanto a Paraíso Pedrês é considerada de crescimento rápido.

Michelan Filho \& Souza (2001) afirmaram que, na produção de frangos de corte, desde 1948 até os dias atuais, o processo de seleção e cruzamentos têm sido intenso, descaracterizando as raças e originando linhagens específicas com características próprias, que, cruzadas entre si, dão origem a um produto híbrido, como as aves Ross.

As aves da linhagem Ross, por serem destinadas a sistema de criação intensivo, têm sido submetidas à seleção para alto ganho de peso à idade precoce (Mccarthy, 1977), de modo que 1 a $2 \%$ do ganho de peso por ano é decorrente do melhoramento genético (Coutinho et al., 2000). 
Tabela 3 - Desdobramento da interação sexo $\times$ linhagem para pesos de carcaça, pernas e carne de pernas em frangos de corte aos 56 dias de idade

Table 3 - Unfolding of sex $\times$ strain interaction for weights of carcass, legs and leg meat in broiler chickens at 56 days of age

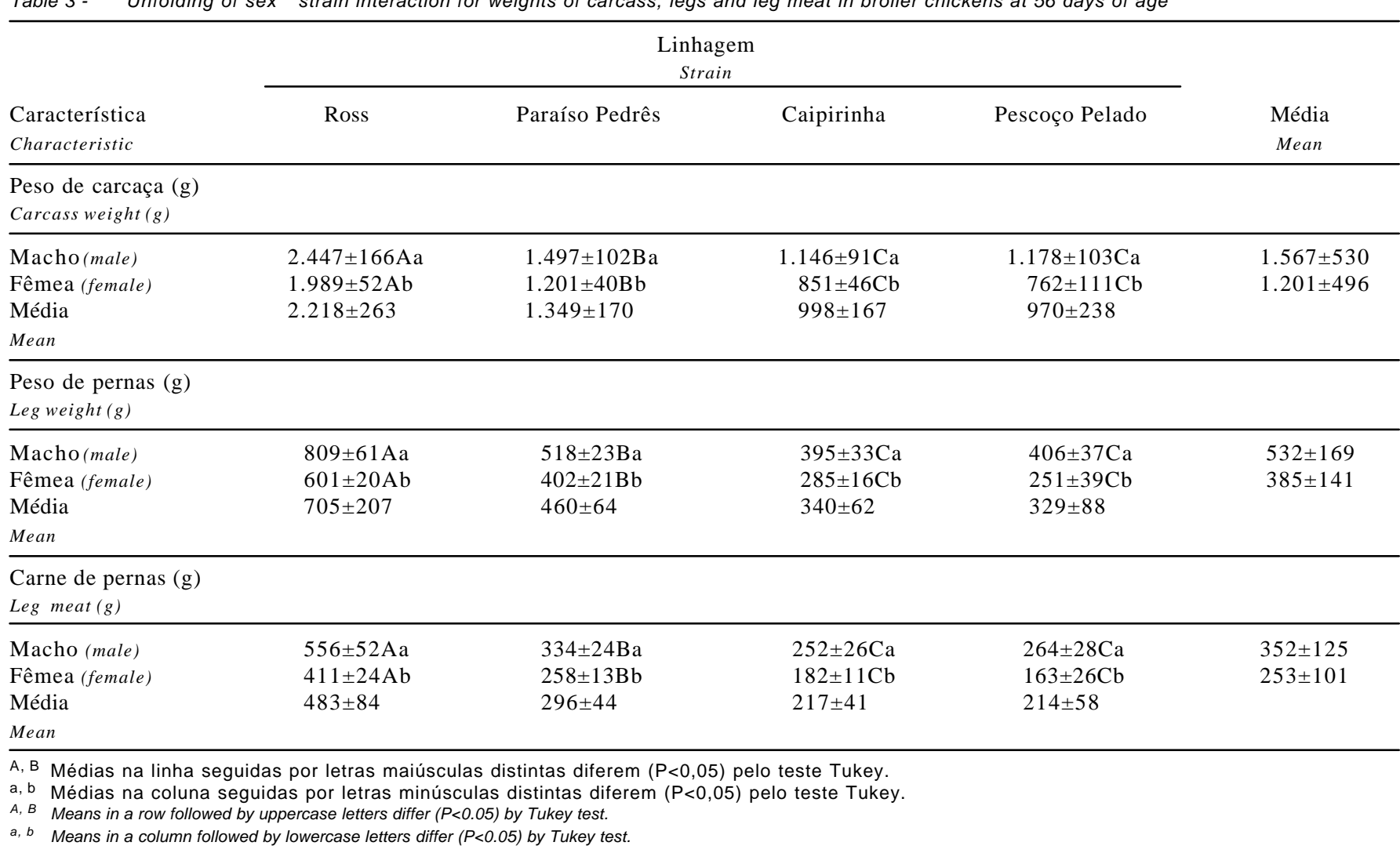

Dentro do fator linhagem, o peso da carcaça foi s empre superior $(\mathrm{P}<0,05)$ nos machos em relação às fêmeas (Tabela 3), o que está de acordo com os resultados observados na indústria e pesquisa, consistindo em uma característica inerente à espécie. Rondelli et al. (2003), ao avaliarem o efeito do sexo no desempenho de duas linhagens comerciais de frangos de corte (Ross e Avian Farm), verificaram maior peso final e ganho de peso nos machos em comparação às fêmeas.

Os resultados dos pesos de carcaça, de pernas e do músculo flexor longo do hálux não foram influenciados ( $P>0.05$ ) pelo sistema de criação (Tabela 2), possivelmente em virtude do curto período em que as aves foram submetidas ao exercício (dos 35 aos 56 dias de idade, 21 dias).

Para a variável peso de pernas, não foi observada interação $(P>0,05)$ sistema de criação $\times$ linhagem e sistema de criação $\times$ sexo. O peso de carne de pernas também não foi influenciado pela interação $(\mathrm{P}>0,05)$ sistema de criação $\times$ linhagem (Tabela 2). Os pesos de pernas e de carne de pernas, no entanto, foram influenciados pela interação $(\mathrm{P}<0,05)$ linhagem $\times$ sexo, conforme o desdobramento é apresentado na Tabela 3. Dentro do fator sexo, a linhagem afetou significativamente $(\mathrm{P}<0,05)$ os rendimentos e pernas e de carne de pernas, pois, tanto para machos como para fêmeas, os pesos de perna e de carne de pernas das aves Ross foram maiores $(\mathrm{P}<0,05)(809 \mathrm{e} 601 ; 556 \mathrm{e} 411 \mathrm{~g})$ que para as da linhagem Paraíso Pedrês (518 e 402; 334 e 258 g). Os pesos de perna e de carne de pernas de machos e fêmeas das linhagens Caipirinha (395 e 285; 252 e 182 g) e Pescoço Pelado (406 e 251; 264 e 163 g) não diferiram e foram inferiores aos das aves Paraíso Pedrês. Em todas as linhagens estudadas, os pesos de pernas e de carne de pernas foram sempre superiores $(\mathrm{P}<0,05)$ nos machos em relação às fêmeas (Tabela 3).

Rondelli et al. (2003), comparando linhagens Ross e Avian Farm, também verificaram que machos machos Ross apresentaram melhores resultados de peso de perna e que não houve diferença entre as fêmeas de ambas as linhagens. Os maiores pesos de pernas e de carne de pernas obtidos nas linhagens de crescimento rápido demonstraram o efeito da maior pressão de seleção nestas linhagens sobre a deposição de massa muscular.

Observou-se interação sistema de criação $\times$ sexo $(\mathrm{P}<0,05)$ para peso de carne de pernas (Tabela 2$)$. Dentro do fator sexo (Tabela 4), somente nos machos houve efeito do sistema de criação $(\mathrm{P}<0,05)$, ressaltando-se que aqueles criados em sistema de confinamento apresentaram maior peso de carne de pernas em comparação aos do sistema de 
Tabela 4 - Desdobramento da interação sexo $\times$ sistema de criação para peso de carne de pernas de frangos de corte aos 56 dias de idade

Table 4 - Unfolding of sex $\times$ breeding system interaction for weight of leg meat of broiler chickens at 56 days of age

\begin{tabular}{llll}
\hline & \multicolumn{2}{c}{$\begin{array}{c}\text { Sistema de criação } \\
\text { Breeding } \text { system }\end{array}$} & \\
\cline { 2 - 3 } Característica & $\begin{array}{c}\text { Confinamento } \\
\text { Confined }\end{array}$ & $\begin{array}{c}\text { Semiconfinamento } \\
\text { Semi-confined }\end{array}$ & $\begin{array}{c}\text { Média } \\
\text { Mean }\end{array}$ \\
\hline Carne de pernas, g & & & \\
Leg meat $(g)$ & & $341 \mathrm{Ba}$ & $352 \pm 125$ \\
\hline Macho (male) & $363 \mathrm{Aa}$ & $257 \mathrm{Ab}$ & $253 \pm 101$ \\
Fêmea (female) & $250 \mathrm{Ab}$ & $299 \pm 122$ & \\
Média (mean) & $307 \pm 123$ & & \\
\hline A, B & & & \\
\hline
\end{tabular}

A, B Médias na linha seguidas por letras maiúsculas distintas diferem $(P<0,05)$ significativamente.

a, b Médias na coluna seguidas por letras minúsculas distintas diferem $(P<0,05)$ significamente.

$A, B$ Means in a row followed by uppercase letters significantly differ $(P<0.05)$.

$a, b$ Means in a column followed by lowercase letters significantly differ $(P<0.05)$.

semiconfinamento. Em ambos os sistemas de criação, o peso de carne de pernas foi superior nos machos $(\mathrm{P}<0,05)$.

Para a variável peso do músculo flexor longo do hálux, não foi observada qualquer interação (Tabela 2). Houve efeito de sexo $(\mathrm{P}<0,05)$, sendo que os machos apresentaram maior peso de músculo (1,67 vs 1,26 g), o que, possivelmente, resultou de fatores intrínsecos da ave, relacionados ao sexo.

Diferenças significativas também foram encontradas entre as linhagens, de modo que as aves Ross apresentaram maior $(\mathrm{P}<0,05)$ peso de músculo flexor longo do hálux (2,34 g), sendo seguidas das aves Paraíso Pedrês $(1,47 \mathrm{~g})$ e Caipirinha e Pescoço Pelado $(1,10$ e 0,99 g, respectivamente), que não diferiram.

$\mathrm{Na}$ Tabela 5 constam os resultados de rendimento de carcaça, pernas, carne de pernas e músculo flexor longo do hálux em relação ao peso vivo antes do abate. Não foi observada interação $(P>0,05)$ nem efeito de sistema de criação sobre as características de rendimento estudadas. Almeida \& Zuber (2000) também não encontraram diferença significativa do sistema de criação para rendimento de carcaça ao trabalharem com frangos de corte tipo colonial.

Para o rendimento de carcaça e de carne de pernas, houve efeito significativo de linhagem. As aves da linhagem Ross, em comparação às demais (que não diferiram), apresentaram maiores $(\mathrm{P}<0,05)$ rendimentos de carcaça e carne de pernas. Não houve efeito de linhagem $(\mathrm{P}>0,05)$ para os rendimentos de pernas e do músculo flexor longo do hálux (Tabela 5). Hellmeister Filho et al. (2002), trabalhando com aves caipiras, também não verificaram diferenças para rendimento de carcaça, pernas e carne de pernas entre linhagens Paraíso Pedrês, Caipirinha e Pescoço Pelado (Label Rouge).
Tabela 5 - Valores médios, em relação ao peso vivo, para os rendimentos (\%) de carcaça $(\mathrm{CA})$, de pernas (PE), de carne de pernas (CPE) e do músculo flexor longo do hálux (MFLH) de frangos de corte aos 56 dias de idade, segundo linhagem, sexo e sistema de criação Table 5 - Mean values of yields of carcass (CA), legs (PE), meat of legs (CPE) and flexor hallucis longus muscle (MFLH) in relation to live weight, of broiler chickens at 56 days of age according to strain, sex and breeding system

\begin{tabular}{|c|c|c|c|c|}
\hline & & $\begin{array}{r}\text { Rendim } \\
\text { Yiel }\end{array}$ & $\begin{array}{l}\%(\%) \\
\%)\end{array}$ & \\
\hline & $\mathrm{CA}$ & PE & CPE & MFLH \\
\hline $\begin{array}{l}\text { Linhagem (L) } \\
\text { Strain }\end{array}$ & 0,01 & $\mathrm{~ns}$ & 0,01 & $\mathrm{~ns}$ \\
\hline $\begin{array}{l}\text { Ross } \\
\text { Paraíso Pedrês } \\
\text { Caipirinha } \\
\text { Pescoço Pelado }\end{array}$ & $\begin{array}{l}72,53 a \\
66,72 b \\
66,06 b \\
66,86 b\end{array}$ & $\begin{array}{l}22,88 \\
22,72 \\
22,46 \\
22,57\end{array}$ & $\begin{array}{l}15,67 \mathrm{a} \\
14,60 \mathrm{~b} \\
14,32 \mathrm{~b} \\
14,65 \mathrm{~b}\end{array}$ & $\begin{array}{l}0,077 \\
0,072 \\
0,073 \\
0,067\end{array}$ \\
\hline $\begin{array}{l}\text { Sexo (S) } \\
\text { Sex } \\
\end{array}$ & $\mathrm{ns}$ & 0,01 & 0,01 & ns \\
\hline $\begin{array}{l}\text { Macho (male) } \\
\text { Fêmea (female) }\end{array}$ & $\begin{array}{l}67,97 \\
67,98\end{array}$ & $\begin{array}{l}23,24 a \\
22,08 b\end{array}$ & $\begin{array}{l}15,19 \mathrm{a} \\
14,41 \mathrm{~b}\end{array}$ & $\begin{array}{l}0,074 \\
0,070\end{array}$ \\
\hline $\begin{array}{l}\text { Sistema de criação }(\mathrm{C}) \\
\text { Breeding system }\end{array}$ & $\mathrm{ns}$ & $\mathrm{ns}$ & $\mathrm{ns}$ & ns \\
\hline $\begin{array}{l}\text { Confinado } \\
\text { Confined } \\
\text { Semi-confinado } \\
\text { Semi-confined } \\
\end{array}$ & $\begin{array}{l}68,14 \\
67,81\end{array}$ & $\begin{array}{l}22,61 \\
22,69\end{array}$ & $\begin{array}{l}14,73 \\
14,86\end{array}$ & 0,073 \\
\hline $\begin{array}{l}\text { Interação } \mathrm{L} \times \mathrm{S} \\
L \times S \text { interaction }\end{array}$ & $\mathrm{ns}$ & $\mathrm{ns}$ & $\mathrm{ns}$ & $\mathrm{ns}$ \\
\hline $\begin{array}{l}\text { Interação } \mathrm{L} \times \mathrm{C} \\
L \times C \text { interaction }\end{array}$ & $\mathrm{ns}$ & $\mathrm{ns}$ & $\mathrm{ns}$ & $\mathrm{ns}$ \\
\hline $\begin{array}{l}\text { Interação } \mathrm{C} \times \mathrm{S} \\
C \times S \text { interaction }\end{array}$ & $\mathrm{ns}$ & $\mathrm{ns}$ & $\mathrm{ns}$ & ns \\
\hline $\begin{array}{l}\text { Interaçãa } \mathrm{L} \times \mathrm{S} \times \mathrm{C} \\
L \times S \times C \text { interaction }\end{array}$ & $\mathrm{ns}$ & $\mathrm{ns}$ & $\mathrm{ns}$ & $\mathrm{ns}$ \\
\hline $\mathrm{CV}(\%)$ & 2,23 & 3,62 & 5,82 & 15,00 \\
\hline
\end{tabular}

a, bMédias na coluna seguidas de letras distintas diferem $(P<0,05)$ pelo teste Tukey.

${ }^{\mathrm{a}, \mathrm{b}}$ Means in a column followed by different letters differ $(P<0.05)$ by Tukey test.

Os rendimentos de pernas e de carne de pernas foram maiores nos machos $(\mathrm{P}<0,05)$, mas não houve efeito de sexo $(\mathrm{P}>0,05)$ sobre os rendimentos de carcaça e do músculo flexor longo do hálux. Almeida \& Zuber (2000), trabalhando com linhagens de frangos coloniais, e Moreira (2003), com linhagens convencionais, também verificaram maior rendimento de pernas para os frangos machos.

A linhagem Ross apresentou maior rendimento de carcaça e de carne de pernas que as demais, observando-se melhor rendimento para os machos, provavelmente em virtude de fatores ligados ao sexo.

Nos cortes do músculo flexor longo do hálux submetidos à reação de m-ATPase após pré-incubação ácida ( $\mathrm{pH}$ 4.5), as fibras do tipo SO reagiram intensamente, as do tipo FG reagiram fracamente e as do tipo FOG reagiram 


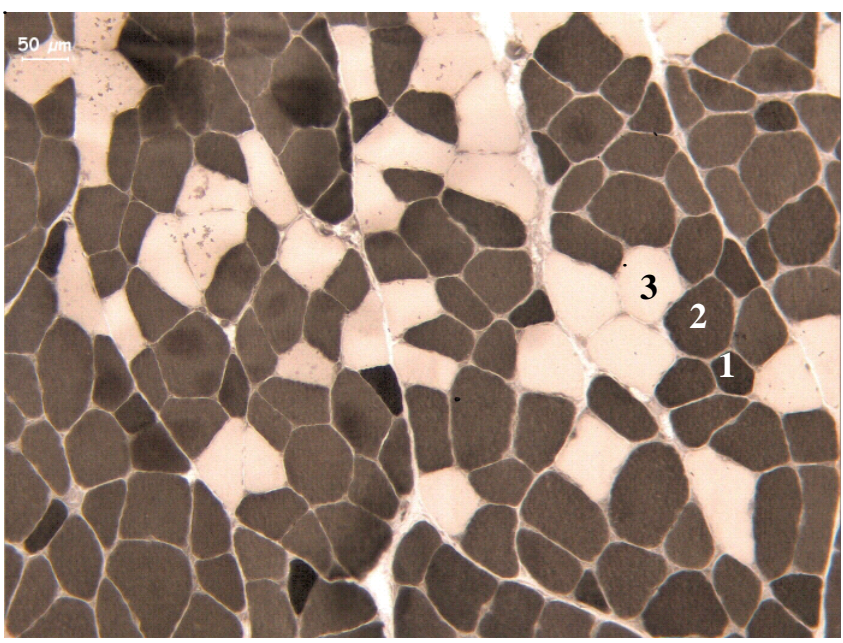

m-ATPase após pré-incubação em pH 4,6 m-ATPase after daily pay-incubation in $\mathrm{pH} 4.6$

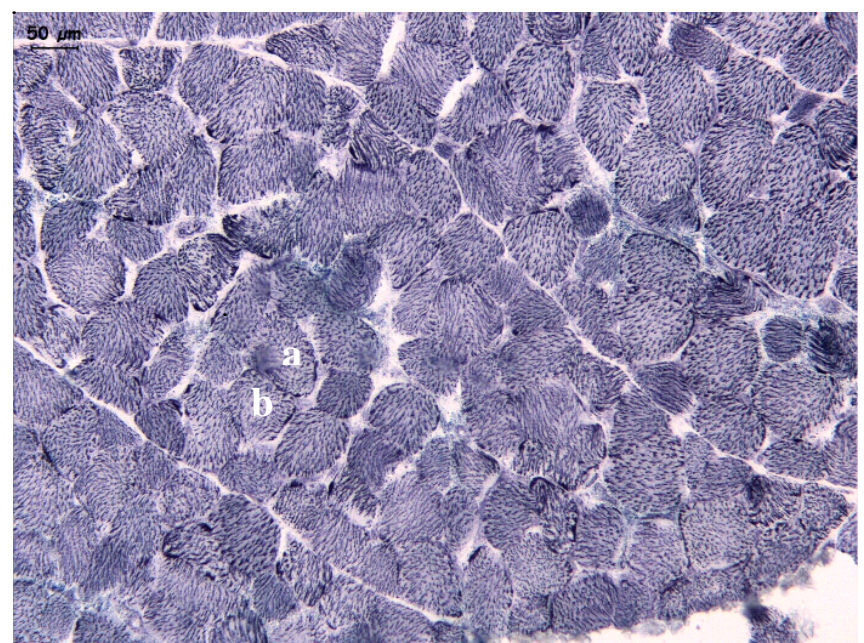

NADH-TR

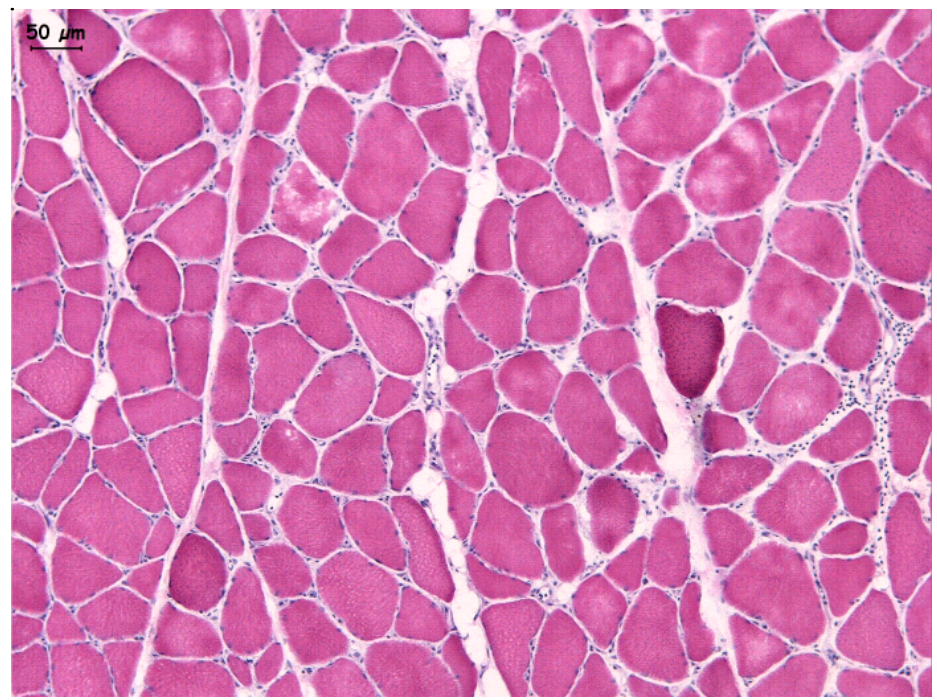

HE

Figura 1 - Cortes tranversais do músculo flexor longo do hálux de frangos de corte aos 56 dias de idade submetidos às reações de m-ATPase após pré-incubação em pH 4,6 e NADH-TR e HE. 1 = SO, 2 = FOG e 3 = FG. a = metabolismo oxidativo intenso e $\mathrm{b}=$ metabolismo oxidativo moderado.

Figure 1 - Cross section of flexor hallucis longus muscle of 56-day-old broilers mATPase, pH 4.6; NADH-TR and HE. $1=S O, 2=$ FOG, $3=$ FG. $a=$ intense oxidative metabolism and $b=$ moderate oxidative metabolism.

moderadamente, com intensidade de coloração intermediária entre FG e SO, permitindo a diferenciação dos três tipos de fibras (Figura 1). Quanto à reação com NADH- TR, as fibras de metabolismo oxidativo (SO e FOG) reagiram mais intensamente em comparação às fibras do tipo glicolítico (FG), que reagiram fracamente, indicando que todas as fibras deste músculo apresentavam predomínio de caráter oxidativo. Não foram observadas alterações na morfologia das fibras musculares do músculo flexor longo do hálux quando submetidas à reação de hematoxilina-eosina (HE).

Como demonstrado na Tabela 6, para área de fibras FG, verificou-se efeito apenas da interação sistema de criação $\times$ sexo $(\mathrm{P}<0,05)$. Houve efeito significativo de linhagem para esta variável, sendo que as aves Ross apresentaram maior $(\mathrm{P}<0,05)$ área de fibras $\mathrm{FG}$, seguidas das Paraíso Pedrês e das linhagens Caipirinha e Pescoço Pelado, que não diferiram entre si. Quanto à área das fibras musculares FOG e SO, não foi observada interação dos fatores estudados, contudo, a linhagem também influenciou significativamente $(\mathrm{P}<0,05)$ estas variáveis. Aves Ross e Paraíso Pedrês apresentaram maiores $(\mathrm{P}<0,05)$ áreas de fibras FOG que as Caipirinha e Pescoço Pelado. Os maiores valores de área $(\mathrm{P}<0,05)$ para as fibras $\mathrm{SO}$ foram encontrados nas aves Ross (Tabela 6), enquanto as demais não diferiram 
Tabela 6 - Valores médios de área $\left(\mathrm{mm}^{2}\right)$ das fibras do tipo FG, FOG e SO do músculo flexor longo do hálux de frangos de corte aos 56 dias de idade, segundo a linhagem, o sexo e o sistema de criação

Table 6 - Mean values of area (ini) of fibers type FG, FOG and SO of the flexor hallucis longus muscle of broiler chickens at 56 days of age according to strain, sex and breeding system

\begin{tabular}{lccc}
\hline & \multicolumn{3}{c}{$\begin{array}{c}\text { Área } \\
\text { Area }\end{array}$} \\
\cline { 2 - 4 } & FG & FOG & SO \\
\hline Linhagem (L) & 0,01 & 0,01 & 0,01 \\
Strain & & & \\
\hline Ross & $7104,51 \mathrm{a}$ & $4915,77 \mathrm{a}$ & $4860,84 \mathrm{a}$ \\
Paraíso Pedrês & $4442,22 \mathrm{~b}$ & $4270,18 \mathrm{a}$ & $2735,20 \mathrm{~b}$ \\
Caipirinha & $3067,20 \mathrm{c}$ & $3308,73 \mathrm{~b}$ & $2677,56 \mathrm{~b}$ \\
Pescoço Pelado & $3062,76 \mathrm{c}$ & $3148,09 \mathrm{~b}$ & $2199,20 \mathrm{~b}$ \\
\hline $\begin{array}{l}\text { Sexo (S) } \\
\text { Sex }\end{array}$ & 0,05 & $\mathrm{~ns}$ & $\mathrm{~ns}$ \\
\hline $\begin{array}{l}\text { Macho } \\
\text { Male }\end{array}$ & 4734,6 & 3699,07 & 2948,39 \\
Fêmea & 4029,7 & 4084,29 & 3228,24 \\
Female & & & \\
\hline
\end{tabular}

\begin{tabular}{|c|c|c|c|}
\hline $\begin{array}{l}\text { Sistema de criação }(\mathrm{C}) \\
\text { Breeding system }\end{array}$ & $\mathrm{ns}$ & $\mathrm{ns}$ & $\mathrm{ns}$ \\
\hline $\begin{array}{l}\text { Confinado } \\
\text { Confined } \\
\text { Semiconfinamento } \\
\text { Semi-confined }\end{array}$ & $\begin{array}{l}4466,29 \\
4597,96\end{array}$ & $\begin{array}{l}4014,99 \\
3778,25\end{array}$ & $\begin{array}{l}3087,65 \\
3093,33\end{array}$ \\
\hline $\begin{array}{l}\text { Interação } \mathrm{L} \times \mathrm{S} \\
L \times S \text { interaction }\end{array}$ & $\mathrm{ns}$ & $\mathrm{ns}$ & $\mathrm{ns}$ \\
\hline $\begin{array}{l}\text { Interação } \mathrm{L} \times \mathrm{C} \\
L \times C \text { interaction } \\
\text { Interação } \mathrm{C} \times \mathrm{S}\end{array}$ & 0,05 & $\mathrm{~ns}$ & $\mathrm{~ns}$ \\
\hline $\begin{array}{l}C \times S \text { interaction } \\
\text { Interação } \mathrm{L} \times \mathrm{S} \times \mathrm{C} \\
L \times S \times C \text { interaction } \\
\mathrm{CV}, \%\end{array}$ & 31,00 & $\begin{array}{c}\text { ns } \\
23,55\end{array}$ & $\begin{array}{c}\mathrm{ns} \\
34,92\end{array}$ \\
\hline
\end{tabular}

para essa característica. Não houve efeito de sistema de criação para área das fibras FG, FOG e SO nem efeito de sexo para área de fibras FOG e SO.

Sartori et al. (1999) também observaram maior área das fibras FG no músculo flexor longo do hálux quando compararam uma linhagem de alto crescimento (Hubbard) a outra de crescimento lento (Pescoço Pelado). Esses autores relataramque a maior massa muscular dos frangos de corte selecionados foi caracterizada pelo aumento da área das fibras glicolíticas em relação àdas fibras oxidativas (FOG + $\mathrm{SO})$. Além disso, nesta pesquisa, as fibras oxidativas também apresentaram maior área nas linhagens de alto crescimento (Ross e Paraíso Pedrês), indicando que a maior pressão de seleção para ganho de peso se manifesta na hipertrofia das fibras musculares.
Tabela 7 - Desdobramento da interação sexo ×sistema de criação para área $\left(\mathrm{mm}^{2}\right)$ de fibras do tipo $F G$ de frangos de corte aos 56 dias de idade

Table 7 - Unfolding of interaction between sex and breeding system for area $\left(\mathrm{mm}^{2}\right)$ of fibers of type FG of broiler chickens at 56 days of age

\begin{tabular}{|c|c|c|c|}
\hline \multirow[b]{2}{*}{$\begin{array}{l}\text { Característica } \\
\text { Characteristic }\end{array}$} & \multicolumn{2}{|c|}{$\begin{array}{l}\text { Sistema de criação } \\
\text { Breeding system }\end{array}$} & \multirow[b]{2}{*}{$\begin{array}{l}\text { Média } \\
\text { Mean }\end{array}$} \\
\hline & $\begin{array}{c}\text { Confinamento } \\
\text { Confined }\end{array}$ & $\begin{array}{l}\text { Semiconfinamento } \\
\text { Semi-confined }\end{array}$ & \\
\hline $\begin{array}{l}\text { Macho } \\
\text { Male }\end{array}$ & $5187,03 \mathrm{Aa}$ & $4.282,17 \mathrm{~B}$ & $\begin{array}{l}4.734,6 \pm \\
2.287,86\end{array}$ \\
\hline $\begin{array}{l}\text { Fêmea } \\
\text { Female }\end{array}$ & $3745,55 b$ & $4.313,76$ & $\begin{array}{l}4.029,7 \pm \\
1.964,69\end{array}$ \\
\hline Média & $4.466,29 \pm$ & $4.597,96 \pm$ & \\
\hline Mean & $2.127,66$ & $2.187,25$ & \\
\hline
\end{tabular}

A, B Médias na linha seguidas por letras maiúsculas distintas diferem significamente $(P<0,05)$.

a, b Médias na coluna seguidas por letras minúsculas distintas diferem significamente $(\mathrm{P}<0,05)$

A, B Means in a row followed by uppercase letters significantly differ $(P<0.05)$.

$a, b$ Means in a column followed by lowercase letters significantly differ $(P<0.05)$.

Dentro do fator sexo, o sistema de criação afetou significativamente a área das fibras do tipo FG, de modo que, nos machoscriados no sistema de confinamento, a área de fibras FG $(\mathrm{P}<0,05)$ foi maior que naqueles do sistema de semiconfinamento (Tabela 7). Esse efeito de sistema de criação não foi observado $(\mathrm{P}>0,05)$ nas fêmeas.

Dentro do sistema de criação, entre as aves criadas em confinamento, verificou-se maior área de fibras FG nas fêmeas $(\mathrm{P}<0,05)$, porém, entre as aves criadas em semiconfinamento, esta diferença entre sexos não foi observada (Tabela 7).

Vários pesquisadores relataram que a seleção para tamanho corporal ou taxa de crescimento rápido em várias espécies animais proporciona sensíveis diferenças na massa muscular em decorrência de alterações no número e no tamanho das fibras musculares atribuídas ao melhoramento genético (Remignon et al., 1994; Fowler et al., 1980).

Quanto ao efeito do exercício, os dados indicam que os machos são mais sensíveis que as fêmeas. A diferença de tamanho da fibra do tipo FG entre machos e fêmeas foi observada somente no sistema de confinamento, o que reforça a afirmativa de que, possivelmente, a menor atividade física das fêmeas e a disponibilidade do alimento ad libitum estejam associadas à maior voracidade de consumo dos machos, o que permite aos machos manifestar seu potencial de crescimento.

$\mathrm{O}$ efeito da linhagem na frequiência das fibras foi verificado somente nas fibras $\mathrm{SO}$, observando-se menor valor para a linhagem Caipirinha (Tabela 8).

A ocorrência de fibras FG, FOG e SO não foi afetada pelo sistema de criação, mas observou-se maior freqüência de 
fibras FG $(\mathrm{P}<0,05)$ nos machos, enquanto, nas fêmeas, houve maior frequiência de fibras FOG (Tabela 8). A ocorrência de fibras SO não foi afetada significativamente ( $P>0,05)$ pelo sexo. A maior freqüência de fibras FG nos machos (Tabela 8) e a maior área de fibras FG dos machos criados em confinamento em relação às fêmeas (Tabela 7) eram esperadas, pois os machos possuem maior massa muscular, comprovando os resultados de peso e rendimentos de carcaça, pernas e carne de pernas (Tabela 3).

Spindler et al. (1980) verificaram que a frequiência e o volume relativo ocupado pelas fibras intermediárias foram relativamente constantes durante o crescimento e, portanto, deve-se considerar que as fibras intermediárias são formas de transição na conversão de fibras vermelhas para brancas. Esses resultados confirmam a hipótese de Ashmore et al.

Tabela 8 - Valores médios de freqüência (\%) das fibras do tipo FG, FOG e SO do músculo flexor longo do hálux de frangos de corte aos 56 dias de idade, segundo a linhagem, o sexo e o sistema de criação

Table 8 - Means values of frequency (\%) of fibers type FG, FOG and SO of flexor hallucis longus muscle broiler of chickens at 56 days of age according to strain, sex and breeding system

\begin{tabular}{|c|c|c|c|}
\hline & \multicolumn{3}{|c|}{$\begin{array}{l}\text { Freqüência (\%) } \\
\text { Frequency }(\%)\end{array}$} \\
\hline & FG & FOG & SO \\
\hline $\begin{array}{l}\text { Linhagem (L) } \\
\text { Strain }\end{array}$ & $\mathrm{ns}$ & $\mathrm{ns}$ & 0,05 \\
\hline $\begin{array}{l}\text { Ross } \\
\text { Paraíso Pedrês } \\
\text { Caipirinha } \\
\text { Pescoço Pelado }\end{array}$ & $\begin{array}{l}15,81 \\
17,18 \\
17,07 \\
12,34\end{array}$ & $\begin{array}{l}59,91 \\
58,12 \\
67,75 \\
62,05\end{array}$ & $\begin{array}{l}24,28 \mathrm{ab} \\
24,70 \mathrm{a} \\
15,18 \mathrm{~b} \\
25,61 \mathrm{a}\end{array}$ \\
\hline $\begin{array}{l}\text { Sexo (S) } \\
\text { Sex }\end{array}$ & 0,01 & 0,05 & $\mathrm{~ns}$ \\
\hline $\begin{array}{l}\text { Macho } \\
\text { Male } \\
\text { Fêmea } \\
\text { Female } \\
\end{array}$ & $18,63 \mathrm{a}$ & $57,85 b$ & $\begin{array}{l}23,52 \\
21,31\end{array}$ \\
\hline $\begin{array}{l}\text { Sistema de criação }(\mathrm{C}) \\
\text { Beeding system }\end{array}$ & $\mathrm{ns}$ & $\mathrm{ns}$ & $\mathrm{ns}$ \\
\hline $\begin{array}{l}\text { Confinado } \\
\text { Confined } \\
\text { Semi-confinado } \\
\text { Semi-confined } \\
\end{array}$ & 15,07 & $\begin{array}{l}61,80 \\
62,10\end{array}$ & $\begin{array}{l}21,90 \\
22,83\end{array}$ \\
\hline $\begin{array}{l}\text { Interação } \mathrm{L} \times \mathrm{S} \\
L \times S \text { interaction }\end{array}$ & $\mathrm{ns}$ & $\mathrm{ns}$ & $\mathrm{ns}$ \\
\hline $\begin{array}{l}\text { Interação } \mathrm{L} \times \mathrm{C} \\
L \times C \text { interaction }\end{array}$ & $\mathrm{ns}$ & $\mathrm{ns}$ & $\mathrm{ns}$ \\
\hline $\begin{array}{l}\text { Interação } \mathrm{C} \times \mathrm{S} \\
C \times \text { S interaction }\end{array}$ & $\mathrm{ns}$ & $\mathrm{ns}$ & $\mathrm{ns}$ \\
\hline $\begin{array}{l}\text { Interação } \mathrm{L} \times \mathrm{S} \times \mathrm{C} \\
L \times S \times C \text { interaction } \\
\mathrm{CV}(\%)\end{array}$ & 21,60 & 15,03 & 24,92 \\
\hline
\end{tabular}

(1972), citados por Spindler et al. (1980), de que o número de fibras brancas aumenta conforme a diferenciação das fibras vermelhas (sem alteração no número total de fibras). Assim, o volume relativo dos diferentes tipos de fibras está relacionado às diferenças acentuadas de massa muscular entre animais de sexo e raça diferentes. Em revisão sobre crescimento muscular, Scheuermann (2004) relatou que, no período pós-eclosão, ocorre alteração na distribuição dos diferentes tipos de fibras musculares no músculo de galináceos, com a modulação de fibras, que se trasformam de fibras de contração rápida para fibras de contração lenta quando submetidas à atividade física.

A maior sensibilidade das fibras do tipo FG a fatores ambientais também foi relatada por Sartori et al. (2003), que observaram redução no tamanho das fibras FG em função de temperatura ambiente fria e de restrição alimentar, fatores relacionados ao menor crescimento e à massa muscular.

Machos, independentemente da linhagem e do sistema de criação, apresentam maior $(\mathrm{P}<0,05)$ freqüência de fibras do tipo FG, possivelmente em virtude da maior taxa metabólica e precocidade, características relacionadas ao sexo.

Houve correlação significativa $(\mathrm{P}<0,05)$ e elevada $(0,93)$ entre o peso do músculo flexor longo do hálux e o peso vivo (Tabela 9). Essa mesma correlação também foi observada por Sartori et al. (2001), com valor um pouco maior (0,97). As áreas de fibras FG, FOG e SO foram positivamente correlacionadas ao peso vivo e ao peso do músculo, indicando contribuição de todas as fibras para

Tabela 9 - Coeficientes de correlação entre peso vivo e dados morfométricos do músculo flexor longo do hálux e dos tipos de fibras em frangos de corte aos 56 dias de idade

Table 9 - Coefficients of correlations between body weigh and morfometric values of flexor hallucis longus muscle and of fiber types of broilers chickens at 56 days of age

\begin{tabular}{lccccccc}
\hline $\begin{array}{l}\text { Variável }^{1} \\
\text { Variable }\end{array}$ & PV & AFG & AFO & ASO & FFG & FFO & FSO \\
\hline PM & $0,93^{*}$ & $0,81^{*}$ & $0,45^{*}$ & $0,64^{*}$ & $0,33^{*}$ & $-0,26$ & 0,089 \\
PV & & $0,77^{*}$ & $0,50^{*}$ & $0,61^{*}$ & 0,25 & $-0,28$ & 0,18 \\
AFG & & & $0,53^{*}$ & $0,60^{*}$ & 0,29 & $-0,31$ & 0,19 \\
AFO & & & & $0,48^{*}$ & 0,06 & $-0,08$ & 0,05 \\
ASO & & & & & $0,17^{*}$ & $-0,10$ & 0,001 \\
FFG & & & & & & $-0,59$ & 0,05 \\
FFO & & & & & & & $-0,83$ \\
FSO & & & & & & & \\
\hline
\end{tabular}

${ }^{1}$ Peso vivo (PV), peso do músculo flexor longo do hálux (PM), área das fibras do tipo $F G(A F G)$, FOG (AFO), SO (ASO) e freqüência das fibras do tipo $F G(F F G)$, FFOG (FFO) e $S O$ (SO).

* Significativo $(P<0,05)$

${ }^{1}$ Body weight (PV), flexor hallucis longus muscle weight (PM), area of fibers type $F G$ $(A F G), F O G(A F O), S O$ (ASO) and frequency fibers of type FG (FFG), FFOG (FFO) and $S O$ (SO).

* Significant $(P<0.05)$ 
o crescimento muscular, porém com maiores valores de correlação para área de fibra FG $(0,77$ para peso vivo e 0,81 para peso do músculo).

Sartori et al. (2001) também encontraram correlações positivas entre o diâmetro das fibras SO, FOG e FG para peso vivo e peso do músculo flexor longo do hálux de frangos de corte e verificaram maiores valores também para as fibras do tipo FG. Esses dados discordam, no entanto, dos obtidos por Knizetova (1993), que observou correlações negativas entre o diâmetro das fibras e o peso do músculo para linhagens de frangos de corte com gene do nanismo ligado ao sexo. De acordo com Rehfeldt (2000), a hipertrofia da fibra muscular pós-natal depende do número total de fibras musculares de um músculo. A taxa de crescimento pós-natal da fibra muscular individual é mais baixa quando há elevado número de fibras no músculo e mais alta quando há menor número de fibras, indicativo de que o número de fibras musculares é inversamente correlacionado à espessura da fibra muscular ao final do período de crescimento intensivo. De fato, Rehfeldt et al. (2000) relataram que coeficientes de correlação genética entre tamanho e número de fibras musculares foram observados para galinhas e suínos, podendo variar de -0,4 a -0,8. Conseqüentemente, a seleção de animais com alta massa muscular, decorrente principalmente da grande proporção de miofibras, resulta em descendentes com baixo número de miofibras.

Neste estudo, verificou-se também correlação positiva $(\mathrm{P}<0,05)$ entre a área dos diferentes tipos de fibras.

\section{Conclusões}

As linhagens Ross-308 e Paraíso Pedrês apresentaram os maiores rendimentos de carcaça e os mais elevados pesos de pernas, de carne de pernas e do músculo flexor longo do hálux, tanto no sistema de confinamento como em semiconfinamento. A maior massa muscular das aves selecionadas para alta taxa de crescimento está relacionada ao aumento na área dos três tipos de fibras musculares ( $\mathrm{SO}$, FOG e FG). Machos possuem maior massa muscular e musculatura mais glicolítica que fêmeas. O sistema de semiconfinamento alterou a composição de fibras musculares esqueléticas dos machos, tornando-a mais oxidativa, contudo, esse efeito não foi observado nas fêmeas.

\section{Literatura Citada}

ASHMORE, C.R.; THOMPKINS, C.; DOERR, L. Postnatal development of muscle fiber types in domestic animals. Journal of Animal Science, v.34, n.1, p.37-41, 1972.
ALMEIDA, A.M.; ZUBER, U. Efeito do sistema de manejo e da alimentação sobre algumas características das carcaças de duas estirpes de frango do tipo "campestre". Veterinária Técnica, v.10, n.5, p.46-50, 2000.

BANKS, W.J. Tecido muscular. In: Histologia veterinária aplicada. 2.ed. São Paulo: Manole, 1992. p.215-236.

BASTIANELLI, D.A. Produção de frangos diferenciados na França: mercado, aspectos organizacionais e regulamentares. In CONFERÊNCIA APINCO DE CIÊNCIA E TECNOLOGIA AVÍCOLAS, 2001, Campinas. Anais... Campinas: Fundação APINCO de Ciência e Tecnologia Avícolas, 2001. p.235-254.

CHAYEN, J.; BITENSKY, L.; BUTCHER, R.G. et al. A guide to practical histochemistry. London: Oliver \& Bould, 1969. $261 \mathrm{p}$.

COUTINHO, L.L.; GABRIEL, J.E.; ALVARES, L.E. Desenvolvimento embrionário da musculatura de frangos: efeito da linhagem e sexo. In: CONFERÊNCIA APINCO DE CIÊNCIA E TECnOlogia AVÍCOLAS, 2000, Campinas. Anais... Campinas: Fundação APINCO de Ciência e Tecnologia Avícolas. 2000, v.2. p.15-32.

DAUNCEY, M.J.; GILMOUR, R.S. Regulatory factors in the control of muscle development. Proceedings of the Nutrition Society, v.55, p.543-559, 1996.

DUBOWITZ, V.; BROOKE, M. Muscle biopsy: a modern approach. London: W.B. Saunders, 1984. 472p.

EVERSOLE, L.S.; STANDISH, S.M. Histochemical demonstration of muscle fibre types. Journal of Histochemistry and Cytochemistry, v. 18, n.8, p.591-593, 1970.

FIGUEIREDO, E.A.P.; AVILA, V.S.; BELLAVER, C. et al. Linhagens e sistema de criação para produção de frangos coloniais. In: CONFERÊNCIA APINCO DE CIÊNCIA E TECNOLOGIA AVÍCOLAS, 2001, Campinas. Anais... Campinas: Fundação APINCO de Ciência e Tecnologia Avícolas, Suplemento 5, p.110, 2003.

FOWLER, S.P.; CAMPION, D.R.; MARKS, H.L. et al. An analysis of skeletal muscle response to selection for rapid growth in Japanese quails (Coturnix Coturnix Japonica). Growth, v.44, n.3, p.235-252, 1980.

GONZALES, E.; SARTORI, J.R. Crescimento e metabolismo muscular. In: MACARI, M.; FURLAN, R. L; GONZALES, E. (Eds.). Fisiologia aviária aplicada a frangos de corte. Jaboticabal: FUNEP/UNESP, 2002. p.279-297.

HELLMEISTER FILHO, P.; MENTEN, J.F.M.; SILVA, M.A.N. et al. Efeito de genótipo e do sistema de criação sobre o desempenho de frangos tipo caipira. Revista Brasileira de Zootecnia, v.32, n.6, 2003

KHASKIYE, A.; RENAUD, D.; LE DOUARIN, G. Effects of electrical stimulation upon post-hatching development of fibre types in normally, innervated fast and slow Latissimus dorsii muscles of the chicken. Biology of the Cell, v.61, n.3, p.163$170,1987$.

KNIZETOVA, H. Effects of the sex-linked dwarf gene (dw) on skeletal muscle cellularity in broiler chickens. British Poultry Science, v.34, n.3, p.479-485, 1993.

McCARTHY, J.C. Growth and poultry meat production. In: POULTRY SCIENCE SYMPOSIUM, 12., 1977, London. Proceedings... London: Butterworths, 1977. p.117.

MENDES, A.A.; GARCIA, E. A.; GONZALES, E. Efeito da linhagem sobre o rendimento de carcaça de frango de corte. Arquivo Brasileiro de Medicina Veterinária e Zootecnia, v.45, p.315-322, 1993.

MICHELAN FILHO, T.; SOUZA, E.M. Formação e características das linhagens atuais de frango. In: CONFERÊNCIA APINCO DE CIÊNCIA E TECNOLOGIA AVÍCOLAS, 2001, Campinas. Anais... Campinas: Fundação APINCO de Ciência e Tecnologia Avícolas, 2001, v.2, p.24-31.

MOREIRA, J.; MENDES, A.A.; ROÇA, R.O et al. Efeito da densidade populacional sobre o desempenho, rendimento de carcaça e qualidade da carne em frangos de corte de diferentes linhagens 
comerciais. Revista Brasileira de Zootecnia, v.33, n.6, p.1506-1519, 2004.

PEARSON, A.M.; YOUNG, R.B. Muscle and meat biochemistry. San Diego: Academic Press, 1989. 457p.

PETER, J.B.; BARNARD, R.J.; EDGERTON, V.R. et al. Metabolic profiles of three fiber types of skeletal muscle in Guinea pig and rabbits. Biochemistry, v.11, n.10, p.2627-2633, 1972.

REHFELDT, C.; FIEDLER, I.; DIETL, G. et al. Myogenesis and postnatal skeletal muscle cell growth as influenced by selection. Livestock Production Science, v.66, p.177-188, 2000.

REMIGNON, H.; LEFAUCHEUR, L.; BLUM, J.C. et al. Effects of divergent selection for body weight on three skeletal muscles characteristics in the chicken. British Poultry Science, v.35, n. 4, p.65-76, 1994.

RONDELLI, S.; MARTINEZ, O.; GARCÍA P.T. Efeito do sexo e parâmetros produtivos, carcass e composição de gordura corporal de duas linhagens comerciais de frangos de corte. Brazilian Journal of Poultry Science, v.5, n.3, p.169-173, 2003.

SANDUSKY, C.L.; HEATH, J.L. Growth characteristics of selected broiler muscles as affected by age and experimental pen design. Poultry Science, v.67, n.11, p.1557-1567, 1988.

SARTORI, J.R.; GONZALES, E.; DAL PAI, V. et al. Tipos de fibras do músculo flexor longo do hálux de frangos de corte machos de diferentes linhagens. Brazilian Journal of Poultry Science, v.1, n.3, p.181-185, 1999.

SARTORI, J.R.; GONZALES, E.; DAL PAI, V. et al. Efeito da temperatura ambiente e da restrição alimentar sobre o desempenho e composição de fibras musculares esqueléticas de frangos de corte. Revista Brasileira de Zootecnia, v.30, n.6, p.1779-1790, 2001.

SARTORI, J.R.; NZALES, E.; MACARI, M. et al. Tipos de fibras no músculo flexor longo do hálux de frangos de corte submetidos ao estresse pelo calor e frio e alimentados em pair-feeding. Revista Brasileira de Zootecnia, v.32, n.4, p.918-925, 2003.
STATISTICAL ANALYSIS SYSTEM - SAS. SAS/STAT. User's guide. Version 6.11. 4.ed. Cary: 1996. v.2, 842p.

SCHEUERMANN, G.N.; BILGILI, S.F.; TUZUN, S. et al. Comparison of chicken genotypes: myofiber number in Pectoralis muscle and myostatin ontogeny. Poultry Science, v.83, p.1404-1412, 2004.

SILVA, M.A.N.; HELLMEISTER FILHO, P.; ROSÁRIO, M.F. et al. Adaptação de linhagens de galinhas para corte ao sistema de criação semi-intensivo. Revista Brasileira de Ciência Avícola, v.4, n.3, p.219-225, 2002.

SOIKE, D.; BERGMANN, V. Performance-dependent health disorders in poultry with special reference to differences in muscle characteristics between layer- and meat type chickens. In: INTERNATIONAL CONFERENCE ON PRODUCTION DISEASES IN FARM ANIMALS, 9., 1995, Berlin. Proceedings... Stuttgart: H. Martins, 1997. p.186.

SPINDLER, A.A.; MATHIAS, M.M.; CRAMER, D.A. Growth changes in bovine muscle fiber types as influenced by breed and Sex. Journal of Food Science, v.45, n.1, p.29-31, 1980.

TAKAHASHI, S.E. Efeito do sistema de criação sobre o desempenho e qualidade da carne de frangos de corte tipo colonial e industrial. Botucatu: Universidade Estadual Paulista, 2003. 64p. Dissertação (Mestrado em Zootecnia) - Universidade Estadual Paulista, 2003. 